\title{
13. Über die Heterogenität der kolloidalen Sole. (Antwort an die Herren G. Quincke und D. Konowalow); von G. Bredig.
}

I. Auf p. 1033, Bd. 9 dieser Annalen schreibt Hr. Quincke: „Die Ansicht Bredigs, die Sole sind äußerst feine mechanische Suspensionen', halte ich für richtig. Aber der Nachweis der zweiphasigen Gebilde fehlt."

Diesen von Hrn. Quincke vermiBten Nachweis glaube ich aber gerade in der von ihm zitierten Monographie (und schon früher) ${ }^{\mathfrak{l}}$ ) ausführlich gegeben $\mathrm{zu}$ haben. Um den Lesern dieser Annalen den Beweis vorzulegen, sei es gestattet, hier einige darauf bezügliche Stellen aus meiner Monographie ${ }^{2}$ ) wörtlich abzudrucken:

„Optische Inhomogenität: Es ist vielfach beobachtet worden, daB kolloidale Gele und Sole, welche im durchfallenden Lichte den Eindruck eines klaren, homogenen Systems machten und selbst unter den besten Mikroskopen keine heterogenen Teilchen entdecken ließen, die größer als die sichtbaren Lichtwellen wären, sich dennoch optisch ${ }^{3}$ ) von gewöhnlichen Kristalloidlösungen durch den sogenannten Tyndallversuch unterscheiden. Ein kolloidales Sol von Eisenhydroxyd ließ z. B. unter dem Mikroskop keine suspendierten Teilchen erkennen, zeigte aber deutlich eine diffuse Zerstreuung eines einfallenden Lichtbündels. ${ }^{4}$ ) Während bei echten fluoreszierenden Kristalloidlösungen das diffus zerstreute Licht unpolarisiert ${ }^{5}$ ) ist, zeigte es sich bei dem Eisenoxydsole und anderen ähnlichen Solen als polarisiert,

1) G. Bredig, Zeitschr. f. angew. Chem. 11. p. 954.1898.

2) G. Bredig, Anorgan. Fermente, Leipzig bei W. Engelmann. 1901. p. 20-22; vgl. auch p. 27 u. 32.

3) E. Verdet, Vorlesungen über die Wellentbeorie des Lichtes 2. p. 403. 1887; Stokes, Phil. Trans. p. 463. 1852; John Strutt (Lord Rayleigh), Phil. Mag. 41. 1871; J. Tyndall, Die Wärme, 4. Aufl. p. 595. 1894.

4) S. E. Linder u. H. Picton, Journ. chem. Soc. 61. p. 152.1892.

5) G. C. Schmidt, Wied. Ann. 60. p. 740. 1897. 
wie man leicht mit Hülfe eines Nicols erkennen kann. Die Polarisation des diffusen Lichtes ist ein Beweis dafür, daB es reflektiertes Licht ist, und daB somit Flüssigkeiten wie das Eisenoxydsol noch Teilchen enthalten, welche zwar zu klein zur mikroskopischen Sichtbarkeit sind, welche jedoch noch groß genug sind im Verhältnis zur Größe der Lichtwellen, um diese $\mathrm{zu}$ polarisieren. Es dürfte dies ${ }^{1}$ ) der empfindlichste Nachweis einer Inhomogenität sein, den wir für solche Medien besitzen. Da wir mit den besten Mikroskopen noch $0,14 \mu$ erkennen können ${ }^{2}$ ), während der Durchmesser einer Wasserstoffmolekel nach der kinetischen Gastheorie etwa $0,00016 \mu$ beträgt $^{3}$ ), so sehen wir, daB die vom Tyndallphänomen noch entdeckten, aher mikroskopisch unsichtbaren, suspendierten Teilchen eines Soles höchstens nur 1000-10000 mal größer, als die sogenannte Molekulardimension sein können.

Elektrische Herstellung von Solen. Es erhebt sich nun die Frage, ob wir kolloidale Sole vielleicht direkt synthetisch erhalten können, wenn wir auf irgend einem Wege einen Stoff mechanisch so fein verteilen, $\mathrm{daB}$ seine Teilchen die obige Größenordnung besitzen. Eine solche Methode zur feinen Zerteilung ist bereits durch Versuche von $\mathrm{O}$. Wiener ${ }^{4}$ ) bewährt worden, welcher zeigte, daß man durch elektrische Zerstäubung einer Silberkathode im Vakuum auf einer Glimmerplatte sogar noch Silberschichten ${ }^{5}$ ) von der Dicke $0,00014 \mu$ erhält, also von der sogenannten Molekulardicke. Ich habe nun gefunden, daß man tatsächlich durch elektrische Kathodenzerstäubung von Metalldrähten unter Wasser kolloidale Sole erhält, wie wir im folgenden sehen werden, sodaß die Ansicht, daß die Sole nur

1) Diese Stelle ist im Original nicht besonders hervorgehoben.

2) Vgl. O. Wiener, Die Erweiterung unserer Sinne, p. 12. Leipzig 1900.

3) Vgl. W. Ostwald, Grundr. d. allgem. Chemie, 3. Aufl. p. 82. 1899.

4) O. Wiener, l. c. p. 14; Wied. Ann. 31. p. 666. 1887.

5) Es wäre von höchstem Interesse für die Theorie des latenten Bildes etc., mit Hülfe der Wienerschen Methoden zu prüfen, ob sich solche dünne Ag-Schichten noch physikalisch entwickeln lassen. Vgl. W. Ostwald, Lehrb. d. allgem. Chem. (2. Aufl) 2. (1) p. 1078; 2. (2) p. 704-784; Zeitschr. f. physik. Chem. 22. p. 289. 1897; R. Abegg, Arch. f. wissenseh. Photogr. 1. p. 15, 109 u. 268. 1899; R. Luther, Areh. f. wissensch. Photogr. 1. p. 274. 1899; K. Schaum, 1. c. 1. p. 139. 1899; G. Bredig, Eders Jahrb. 13. p. 357. 1899. 
änßerst feine mechanische Suspensionen sind, durch diese synthetische Darstellungsmethode eine neue Stütze erhält."

Ebenso wird auf p. 27 u. 32 l. c. von mir ausdrücklich die optische Inhomogenität der durch Zerstäubung erhaltenen Gold- und Silbersole, also ihre Natur als ,zweiphasiges Gebilde", in Übereinstimmung mit ihren übrigen Eigenschaften nachgewiesen. Der von mir im AnschluB an Linder und Picton (l. c.) u. a. gelieferte optische Nachweis der Inhomogenität der Goldsole scheint auch neuerdings schlagende Bestätigung durch die soeben erschienene interessante Arbeit von Siedentopf u. Zsigmond $\mathrm{y}^{1}$ ) zu erfahren, wenn die darin beschriebene Methode zum Nachweis ultramikroskopischer Teilchen ohne Einwand bleibt. Hr. Zsigmondy hat übrigens früher meine Ansicht von der heterogenen Struktur der Sole nicht geteilt ${ }^{2}$ ), während er jetzt sogar mit Hrn. Siedentopf die Größe dieser darin heterogen suspendierten Teilchen bestimmt hat.

$\mathrm{DaB}$ es stetige Übergänge von den heterogenen Solen zu den homogenen echten Lösungen (wenigstens nach dem Stande unserer experimentellen Grenzen) geben kann, wie Picton und Linder sowie Lobry de Bruyn ${ }^{3}$ ) behaupten, habe ich bisher nie bestritten, fand aber das einzige sichere Kriterium der Unterscheidung bez. die experimentelle Grenze zwischen Homogen und Heterogen eben nur in dem Versuche nach Stokes-Tyndall mit dem Nicolschen Prisma, wenn man nicht die Beziehungen von Gleichgewichten zur Oberflächengröße zu Hülfe nehmen will ${ }^{4}$ ) oder kann.

Meine Auffassung der Koagulation als einer Erscheinung, die mit den kapillarelektrischen Eigenschaften in Zusammenhang

1) H. Siedentopf u. R. Zsigmondy, Ann. d. Phys. 10. p. 1. 1900.

2) Vgl. R. Zsigmondy, Zeitschr. f. physik. Chem. 33. p. 71. 1900.

3) Lobry de Bruýn, Recueil des Travaux chim. des Pays-Bas 19. p. 251.1900 .

4) Vgl. W. Ostwald, Zeitschr. f. physik. Chem. 34. p. 495. 1900; G. Bredig, Eders Jahrb. d. Photogr. 13. p. 357. 1899; A. Mittasch, Zeitschr. f. physik. Chem. 40. p. 38. 1902; G. Hulett, ebenda 37. p. 385. 1901. Bei dieser Gelegenheit muB ich jede Identität oder Solidarität mit dem wunderlichen anonymen Energetiker Quinckes auf p. 1019-1020 ausdrücklich ablehnen, da ein solches Mißverständnis durch die Darstellung $Q$ uinckes nicht ausgeschlossen wird. 
steht, wird, wie ich l. c. gezeigt habe, geradezu notwendig, wenn man überhaupt den auch durch Quinckes bekannte ältere Versuche begründeten Satz anerkennt, daß sich in einem "zweiphasigen Gebilde" eine elektrische Potentialdifferenz an der Grenze zwischen den beiden heterogenen Medien ausbildet, und wenn man die Koagulation als eine Ausflockung unter Verkleinerung dieser gemeinsamen Oberfläche auffaBt, wie ich es getan habe. Höchstens wäre mit Rücksicht auf neuere Untersuchungen die Spezialbedingung von Helmholtz fallen zu lassen, daB gerade zu der Potentialdifferenz Null der beiden Medien das Maximum ihrer gegenseitigen Oberflächenspannung und daher nach meiner und Hardys Annahme auch das Maximum der Instabilität ihres Soles gehört.

Hr. Quincke bebauptet ferner, in meinen Betrachtungen bleibe „der Einfluß der Oberflächenspannung an der Grenze der zweiphasigen Gebilde unberücksichtigt"s. Demgegenüber sei erwähnt, daß ich in meiner Monographie doch als wesentliches Moment bei Koagulation von Solen gerade den Einfluß der Oberflächenspannung in Verbindung mit der Potentialdifferenz eingeführt habe. Vgl. 1. c. p. 14, 16 u. 17.

Es. wird mir ferner von Hrn. Quincke vorgeworfen, „den festen oder flüssigen Aggregatzustand der schwebenden Teilchen“ nicht berücksichtigt $\mathrm{zu}$ haben. Auch in den ausgedehnten Arbeiten meines verehrten Kritikers habe ich bisher keinerlei Entscheidung der Frage gefunden, ob die mikroskopisch unsichtbaren suspendierten Teilchen eines Soles flüssig oder fest sind.

II. Auf p. 384, Bd. 10 dieser Annalen schreibt Hr. Konowalow, nachdem er seine Dampfdruckkurven im kritischen Gebiete der Lösungen diskutiert hat:

„Die angeführten Tatsachen beweisen, daß wir es sowohl in Flüssigkeits- als auch in kolloidalen Lösungen mit ein und derselben Bedingung $\mathrm{zu}$ tun haben: eine deutliche Opaleszenz ist denjenigen Lösungen eigen, welche ihren Dampfdruck bei Veränderung der Konzentration kaum verändern, also Lösungen, in welchen durch den unbedeutendsten Arbeitsaufwand große Veränderungen der Konzentration hervorgerufen werden könneu.

Zum Vergleich sei hier ein Passus aus meiner von ihm nur ganz beiläufig an anderer Stelle erwähnten älteren Monographie wörtlich abgedruckt (l. c. p. 10): 
„Arbeit bei der Entfernung des Lösungsmittels: Ein schon viel schärferes Kennzeichen der kolloidalen Gemische ist die Tatsache, daß die Arbeit, welche nötig ist, um ihre Komponenten, also „Lösungsmittel" und „Gelöstes", voneinander zu trennen, einen im Vergleich zu Kristalloidlösungen auffallend kleinen Betrag hat. Bekanntlich besitzen wir in dem osmotischen Drucke, der relativen Dampfdruckverminderung und der Gefrierpunktserniedrigung ein direktes $\mathrm{MaB}$ für diese Arbeit."

Hr. Konowalow fährt ferner fort:

„Mit Hülfe der Tyndallschen Methode kann man sich leicht davon überzeugen, daß auch in Flüssigkeitslösungen das reflektierte Licht in einer zur Richtung des durchgehenden Strahles senkrechten Ebene polarisiert ist, ganz wie in kolloidalen Lösungen."

Hr. Konowalow vergibt aber hinzuzufügen, daB bereits Hr. Friedländer ${ }^{1}$ vor ihm diese auf meine Veranlassung festgestellte Tatsache angibt, daB die kritisch getrübten „Flüssigkeitslösungen" mit den anderen trüben Medien und Kolloiden die Eigenschaft gemeinsam haben, „das reflektierte Licht in der zum Strahl senkrechten Ebene zu polarisieren."

Die von Friedländer konstatierte Stabilität dieser Trübung kritischer Lösungen im elektrischen Potentialgefälle sowie die Konstanz und quantitative photometrische Reproduzierbarkeit der Trübung spricht gegen die Ansicht Konowalows, daß das Hauptmoment zur Bildung derselben die zufälligen Staubpartikeln sind, die ja in ihrer Menge schwanken und im Potentialgefälle wandern mäßten.

Heidelberg, Januar 1903.

1) J. Friedländer, Zeitschr. f. physik. Chem. 38. p. 438. 1901.

(Eingegangen 30. Januar 1903.) 